\title{
DYADIC GREEN'S FUNCTIONS FOR CYLINDRICAL WAVEGUIDES WITH MOVING MEDIA
}

\author{
C. F. STUBENRAUCH and C-T. TAI \\ The University of Michigan Radiation Laboratory \\ Department of Electrical Engineering \\ Ann Arbor (Michigan 48108) USA
}

\begin{abstract}
The dyadic Green's function for cylindrical waveguides of circular or rectangular cross section with a moving, isotropic, homogeneous medium is developed using the method of eigenfunction expansion. The orthogonality properties of the vector mode functions are discussed. In contrast to waveguides with a stationary medium, it is seen that the normalization factor in the case of the $E$ mode introduces a pole in the integral representation for the Green's function which must be excluded from the integration contour.
\end{abstract}

\section{$\S 1$. Introduction}

The solution for the modes in a cylindrical waveguide containing a moving isotropic homogeneous medium is well known and has been discussed by several authors in recent years [1-6]. Problems involving forced excitations are easily solved if the dyadic Green's function for the particular system is known. Seto [7] has given a formulation for the dyadic Green's function in eigenfunction form. It is our purpose here to present an alternate and more compact derivation by extending the method of $\mathrm{Ohm}$ and Rayleigh to the wave equation satisfied by the dyadic Green's function. The results so obtained for the rectangular and circular waveguide appear to be more explicit than those obtained by Seto. The same method can be readily applied to elliptical waveguides if so desired.

\section{$\S 2$. Development of the theory}

For a moving medium the constitutive relations can be put in compact form [8],

$$
\bar{B}=\mu \overline{\bar{\alpha}} \cdot \bar{H}-\overline{\boldsymbol{\Omega}} \times \overline{\boldsymbol{E}}
$$




$$
\overline{\boldsymbol{D}}=\varepsilon \overline{\overline{\boldsymbol{\alpha}}} \cdot \overline{\boldsymbol{E}}+\overline{\boldsymbol{\Omega}} \times \overline{\boldsymbol{H}}
$$

where $\varepsilon$ and $\mu$ are permittivity and permeability respectively of the medium at rest

$$
\overline{\boldsymbol{\Omega}}=\Omega \hat{z}=\frac{n^{2}-1}{1-n^{2} \beta^{2}} \frac{\overline{\boldsymbol{v}}}{c^{2}}
$$

$\overline{\boldsymbol{v}}=v \hat{\mathbf{z}}$, velocity of the moving medium

$$
c=\left(\mu_{0} \varepsilon_{0}\right)^{-\frac{1}{2}}
$$$$
n=\left(\frac{\mu \varepsilon}{\mu_{0} \varepsilon_{0}}\right)^{\frac{1}{2}} \text {, index of refraction of the medium at rest }
$$$$
\beta=\frac{v}{c}
$$$$
\overline{\boldsymbol{\alpha}}=a(\hat{\boldsymbol{x}} \hat{\boldsymbol{x}}+\hat{\boldsymbol{y}} \hat{\boldsymbol{y}})+\hat{z} \hat{z}
$$$$
a=\frac{1-\beta^{2}}{1-n^{2} \beta^{2}} \text {. }
$$

It is assumed that the velocity of the moving medium is constant, otherwise the constitutive relations described by (1) and (2) will no longer be valid. We note that for $v=0$ or $n=1$ the constitutive relations reduce to the familiar ones for free space. Limiting ourselves to harmonically oscillating fields $\left(\mathrm{e}^{-\mathrm{i} \omega t}\right)$ and substituting (1) and (2) into Maxwell's equations gives

$$
\begin{gathered}
\nabla \times \overline{\boldsymbol{E}}=\mathrm{i} \omega(\mu \overline{\overline{\boldsymbol{\alpha}}} \cdot \overline{\boldsymbol{H}}-\overline{\boldsymbol{\Omega}} \times \overline{\boldsymbol{E}}) \\
\nabla \times \overline{\boldsymbol{H}}=\overline{\boldsymbol{J}}-\mathrm{i} \omega(\varepsilon \overline{\boldsymbol{\alpha}} \cdot \overline{\boldsymbol{E}}+\overline{\boldsymbol{\Omega}} \times \overline{\boldsymbol{H}}) .
\end{gathered}
$$

Two auxiliary field vectors $\overline{\boldsymbol{E}}^{(b)}$ and $\overline{\boldsymbol{H}}^{(b)}$ are introduced such that

$$
\begin{aligned}
& \overline{\boldsymbol{E}}=\mathrm{e}^{-\mathrm{i} \omega \boldsymbol{\Omega} z \overline{\overline{\boldsymbol{b}}} \cdot \overline{\boldsymbol{E}}^{(b)}} \\
& \overline{\boldsymbol{H}}=\mathrm{e}^{-\mathrm{i} \boldsymbol{\theta} \boldsymbol{\Omega} z} \overline{\overline{\boldsymbol{b}}} \cdot \overline{\boldsymbol{H}}^{(b)}
\end{aligned}
$$

where

$$
\overline{\overline{\boldsymbol{b}}}=\frac{1}{a}(\hat{\boldsymbol{x}} \hat{\boldsymbol{x}}+\hat{\boldsymbol{y}} \hat{\boldsymbol{y}})+\hat{\mathbf{z}} \hat{\boldsymbol{z}} \text { or } \overline{\overline{\boldsymbol{b}}} \cdot \overline{\overline{\boldsymbol{\alpha}}}=\overline{\overline{\boldsymbol{I}}} .
$$

Substituting these into (3) and (4) we obtain the following equations for the reduced fields $\overline{\boldsymbol{E}}^{(b)}$ and $\overline{\boldsymbol{H}}^{(b)}$ :

$$
\boldsymbol{\nabla} \times\left(\overline{\overline{\boldsymbol{b}}} \cdot \overline{\boldsymbol{E}}^{(b)}\right)=i \omega \mu \overline{\boldsymbol{H}}^{(b)}
$$




$$
\nabla \times\left(\overline{\overline{\boldsymbol{b}}} \cdot \overline{\boldsymbol{H}}^{(b)}\right)=\overline{\boldsymbol{J}} \mathrm{e}^{\mathrm{i} \omega \Omega z}-\mathrm{i} \omega \varepsilon \overline{\boldsymbol{E}}^{(b)} .
$$

It is to be noted that the auxiliary fields $\overline{\boldsymbol{E}}^{(b)}$ and $\overline{\boldsymbol{H}}^{(b)}$ are not the same as the auxiliary fields introduced in a previous paper [8]. For analytical reasons we found it more convenient to deal with the two auxiliary fields as defined by (5) and (6). Elimination of $\overline{\boldsymbol{H}}^{(b)}$ between the two equations yields the wave equation

$$
\boldsymbol{\nabla} \times\left[\overline{\overline{\boldsymbol{b}}} \cdot \boldsymbol{V} \times\left(\overline{\overline{\boldsymbol{b}}} \cdot \overline{\boldsymbol{E}^{(b)}}\right)\right]-k^{2} \overline{\boldsymbol{E}}^{(b)}=\mathrm{i} \omega \mu \overline{\boldsymbol{J}} \mathrm{e}^{\mathrm{i} \omega \Omega z}
$$

where $k^{2}=\omega^{2} \mu \varepsilon$ the free space wave number. In view of the structure of (9) we introduce the dyadic Green's function $\overline{\overline{\mathbf{G}}}(b)$ which satisfies the wave equation

$$
\boldsymbol{\nabla} \times \overline{\overline{\boldsymbol{b}}} \cdot\left[\boldsymbol{\nabla} \times\left(\overline{\overline{\boldsymbol{b}}} \cdot \overline{\overline{\boldsymbol{G}}}^{(b)}\right)\right]-k^{2} \overline{\overline{\boldsymbol{G}}}(b)=\overline{\overline{\boldsymbol{I}}} \delta\left(\overline{\boldsymbol{R}}-\overline{\boldsymbol{R}}^{\prime}\right) .
$$

For waveguides made of perfectly conducting walls the function must satisfy the Dirichlet condition

on the walls.

$$
\hat{\boldsymbol{n}} \times \overline{\overline{\boldsymbol{G}}}^{(b)}\left(\overline{\boldsymbol{R}} \mid \overline{\boldsymbol{R}}^{\prime}\right)=0
$$

Applying the vector Green's theorem to (9) and (10) and employing the symmetry properties of dyadic Green's functions, it can be shown that the electric field excited by a current $\overline{\boldsymbol{J}}_{\text {or }}$ an aperture field $\overline{\boldsymbol{E}_{\mathrm{a}}}$ is given by

$$
\begin{aligned}
& \overline{\boldsymbol{E}}(R)=i \omega \mu_{\mathrm{o}} \iint_{\mathbf{V}} \overline{\overline{\boldsymbol{b}}} \cdot \overline{\overline{\boldsymbol{G}}}(b)\left(\overline{\boldsymbol{R}} \mid \overline{\boldsymbol{R}}^{\prime}\right) \cdot \overline{\boldsymbol{J}}\left(\overline{\boldsymbol{R}}^{\prime}\right) \mathrm{e}^{-\mathbf{i} \omega \Omega\left(z-z^{\prime}\right)} \mathrm{d} v^{\prime}+ \\
& \quad+\iint_{\mathbf{A}} \overline{\overline{\boldsymbol{b}}} \cdot \boldsymbol{V} \times\left[\overline{\overline{\boldsymbol{b}}} \overline{\overline{\boldsymbol{G}}}\left(\overline{\overline{\boldsymbol{G}}}\left(\overline{\boldsymbol{R}} \mid \overline{\boldsymbol{R}^{\prime}}\right)\right] \cdot\left[\hat{\boldsymbol{n}} \times \overline{\boldsymbol{E}}_{\mathrm{a}}\left(\overline{\boldsymbol{R}}^{\prime}\right)\right] \mathrm{e}^{-\mathrm{i} \omega \Omega\left(z-z^{\prime}\right)} \mathrm{d} s^{\prime} .\right.
\end{aligned}
$$

To solve for the Green's function $\overline{\overline{\boldsymbol{G}}}^{(b)}$ we will use the method of Ohm and Rayleigh (eigenfunction expansion) as described by Sommerfeld [9] but extend it to the vector case. The advantage of this method is that it allows us to by-pass the determination of the excitation coefficient by a tedious consideration of the discontinuity due to the singular source. We begin by finding a solution to the homogeneous vector wave equation corresponding to (10), that is,

$$
\boldsymbol{\nabla} \times[\overline{\overline{\boldsymbol{b}}} \cdot \boldsymbol{\nabla} \times(\overline{\overline{\boldsymbol{b}}} \cdot \overline{\boldsymbol{F}})]-\boldsymbol{\kappa}^{2} \overline{\boldsymbol{F}}=0
$$

where $\kappa$ is a constant to be determined by the eigenvalues. The 
vector wave functions which are solutions to (12) can be written in the form

$$
\overline{\boldsymbol{M}}_{\mathrm{e}}(h)=\boldsymbol{V} \times\left[\psi_{\mathrm{g}}(h) \hat{\mathbf{z}}\right]
$$

and

$$
\overline{\boldsymbol{N}}_{\mathbf{\mathrm { o }}}(h)=\frac{1}{\kappa} \boldsymbol{\nabla} \times\left[\overline{\overline{\boldsymbol{b}}} \cdot \overline{\boldsymbol{M}}_{\mathbf{\mathrm { e }}}(h)\right]=\frac{1}{\kappa a} \boldsymbol{\nabla} \times \boldsymbol{\nabla} \times\left[\psi_{\mathbf{0}}(h) \hat{\mathbf{z}}\right]
$$

where $\psi_{\mathrm{e}}(h)$ is a solution of

$$
\nabla_{t}^{2} \psi_{\mathrm{o}}+\frac{1}{a} \frac{\partial^{2} \psi_{\mathrm{e}}}{\partial z^{2}}+\kappa^{2} a \psi_{\mathrm{e}}=0
$$

The significance of the subscripts $t$ will be explained later. We note that the vector wave functions satisfy the mutual relations

$$
\begin{aligned}
& \nabla \times\left(\overline{\bar{b}} \cdot \overline{\boldsymbol{M}}_{\mathrm{o}}\right)=\kappa{\overline{N_{\mathrm{e}}}}_{\mathrm{o}} \\
& \nabla \times\left(\overline{\bar{b}} \cdot{\overline{N_{\mathrm{e}}}}_{\mathrm{o}}\right)=\kappa \overline{\boldsymbol{M}}_{\mathrm{o}}
\end{aligned}
$$

and that $\bar{M}$ functions correspond to $H$-modes and $\bar{N}$ functions correspond to $E$-modes if these functions are used to represent the electric field.

\section{§ 3. Rectangular waveguide}

We consider a rectangular waveguide bounded by perfectly conducting walls at $x=0$ and $x=x_{0}$ and at $y=0$ and $y=y_{0}$. We assume that the medium inside the guide is moving in the $z$ direction corresponding to the longitudinal axis of the guide. The eigenfunctions which satisfy (15) are given by

$$
\psi_{\mathrm{o} m n}(h)=\left\{\begin{array}{c}
\cos \\
\sin
\end{array}\right\} \frac{m \pi x}{x_{0}}\left\{\begin{array}{c}
\cos \\
\sin
\end{array}\right\} \frac{n \pi y}{y_{0}} \mathrm{e}^{\mathrm{i} h z}
$$

where ${ }_{0}^{\mathrm{e}}$ indicates that the corresponding trigonometric functions are either even or odd, and

$$
\begin{gathered}
h^{2}=\kappa^{2} a^{2}-a k_{c}^{2} \\
k_{c}^{2}=\left(\frac{m \pi}{x_{0}}\right)^{2}+\left(\frac{n \pi}{y_{0}}\right)^{2} .
\end{gathered}
$$

The atplication of the boundary conditions $\hat{\boldsymbol{n}} \times \overline{\boldsymbol{M}}=0$ and $\hat{\boldsymbol{n}} \times \overline{\boldsymbol{N}}=$ $=0$ ap the guide walls gives the following permissible vector wave 
functions for the eigenfunction expansion of the dyadic Green's function under consideration.

$$
\begin{gathered}
\overline{\boldsymbol{M}}_{\mathrm{e} m n}(h)=\boldsymbol{\nabla} \times\left[\psi_{\mathrm{e} m n}(h) \hat{\boldsymbol{z}}\right] \\
\overline{\boldsymbol{N}}_{\mathrm{o} m n}(h)=\frac{1}{\boldsymbol{\kappa} a} \boldsymbol{\nabla} \times \boldsymbol{\nabla} \times\left[\psi_{\mathrm{o} m n}(h) \hat{\mathbf{z}}\right] .
\end{gathered}
$$

These two sets of vector wave functions exhibit the following orthogonality properties:

$$
\begin{gathered}
\iiint \overline{\boldsymbol{M}}_{\mathrm{e} m n}(h) \cdot \overline{\boldsymbol{N}}_{\mathrm{o} m^{\prime} n^{\prime}}\left(-h^{\prime}\right) \mathrm{d} v=0 \\
\iiint \overline{\boldsymbol{M}}_{\mathrm{e} m n}(h) \cdot \overline{\boldsymbol{M}}_{\mathrm{e} m^{\prime} n^{\prime}}\left(\dot{-} h^{\prime}\right) \mathrm{d} v= \\
=\left\{\begin{array}{l}
0 ; \quad m \neq m^{\prime} \text { or } n \neq n^{\prime} \\
\frac{1+\delta_{0}}{2} \pi k_{c}^{2} x_{0} y_{0} \delta\left(h-h^{\prime}\right) ; \quad m=m^{\prime}, n=n^{\prime}
\end{array}\right.
\end{gathered}
$$

where

also

$$
\delta_{0}= \begin{cases}0 ; & m, n \neq 0 \\ 1 & m=0 \text { or } n=0\end{cases}
$$

$$
\begin{aligned}
& \iiint \bar{N}_{\mathrm{o} m n}(h) \cdot \bar{N}_{\mathrm{o} m^{\prime} n^{\prime}}\left(-h^{\prime}\right) \mathrm{d} v= \\
& \quad=\left\{\begin{array}{l}
0 ; \quad m \neq m^{\prime} \text { or } n \neq n^{\prime} \\
\frac{\pi}{2} k_{c}^{2} x_{0} y_{0} \frac{h^{2}+k_{c}^{2}}{h^{2}+a k_{c}^{2}} \delta\left(h-h^{\prime}\right) ; \quad m=m^{\prime}, n=n^{\prime} .
\end{array}\right.
\end{aligned}
$$

Following the method of $\mathrm{Ohm}$ and Rayleigh we proceed to expand the dyadic Green's function in terms of the vector wave function starting with an expansion of the dyadic delta function

$$
\overline{\overline{\boldsymbol{I}}} \delta\left(\overline{\boldsymbol{R}}-\overline{\boldsymbol{R}}^{\prime}\right)=\int_{\mathrm{C}_{+} \text {or } \mathrm{C}_{-}} \mathrm{d} h \sum_{m} \sum_{n}\left[\overline{\boldsymbol{M}}_{\mathrm{e} m n}(h) \overline{\boldsymbol{A}}_{\mathrm{e} m n}(h)+\overline{\boldsymbol{N}}_{\mathrm{o} m n}(h) \overline{\boldsymbol{B}}_{\mathrm{o} m n}(h)\right]
$$

where $\mathrm{C}_{+}$and $\mathrm{C}_{-}$-are illustrated in Fig. 1. It will be shown later why we choose the particular contours shown in the figure where $\pm \mathrm{i} k_{c}$ denotes two poles which are characteristic of the integrand. 


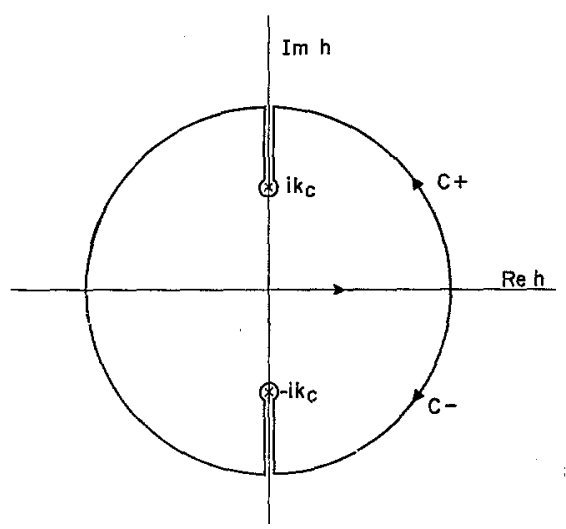

Fig. 1. Contour of Integration.

By taking an anterior scalar product of $(22)$ with $\overline{\boldsymbol{M}}_{\mathrm{e} m^{\prime} n^{\prime}\left(--h^{\prime}\right)}$ and integrating the result over the waveguide volume we can determine $\overline{\boldsymbol{A}}_{\mathrm{emn} n}(h)$ as a result of the orthogonality relations stated by (19) and (20). Solving in like fashion for $\overline{\boldsymbol{B}}_{\mathrm{o} m n}(h)$ we find that

$$
\begin{gathered}
\overline{\boldsymbol{A}}_{\mathrm{e} m n}(h)=\frac{2-\delta_{0}}{\pi x_{0} y_{0} k_{c}^{2}} \overline{\boldsymbol{M}}_{\mathrm{e} m n}^{\prime}(-h) \\
\overline{\boldsymbol{B}}_{\mathrm{o} m n}(h)=\frac{2-\delta_{0}}{\pi x_{0} y_{0} k_{\mathrm{c}}^{2}} \frac{h^{2}+a k_{c}^{2}}{h^{2}+k_{c}^{2}} \overline{\boldsymbol{N}}_{\mathrm{o} m n}^{\prime}(-h)
\end{gathered}
$$

where the prime on $\bar{M}$ and $\bar{N}$ indicate that they are functions of the primed coordinates $x^{\prime}, y^{\prime}$ and $z^{\prime}$.

It is observed that the expression for $\overline{\boldsymbol{B}}_{\text {omn }}(h)$ contains poles at $h= \pm \mathbf{i} k_{c}$. If the contour in the $h$-plane does not exclude these poles it would give rise to a term of the form $\mathrm{e}^{-k_{c}\left|z-z^{\prime}\right|}$ which clearly cannot be part of an expression for a delta function since it does not vanish for $z=z^{\prime}$. In fact, it does not satisfy the homogeneous wave equation. Thus the contour of integration so deformed is the proper contour to be used in this formulation.

Once the expansion of the dyadic delta function is known, we can expand the Green's function in a similar manner, that is

$$
\overline{\overline{\boldsymbol{G}}}(b)\left(\overline{\boldsymbol{R}} \mid \overline{\boldsymbol{R}}^{\prime}\right)=\frac{1}{\pi x_{0} y_{0}} \int_{\mathrm{C}_{+} \text {or }_{\mathrm{C}_{-}}} \mathrm{d} h \sum_{m} \sum_{n} \frac{2-\boldsymbol{\delta}_{0}}{k_{c}^{2}} \times
$$




$$
\times\left\{\alpha \overline{\boldsymbol{M}}_{\mathrm{e} m n}(h) \overline{\boldsymbol{M}}_{\mathrm{e} m n}^{\prime}(-h)+\beta \frac{h^{2}+a k_{c}^{2}}{h^{2}+k_{c}^{2}} \overline{\boldsymbol{N}}_{\mathrm{o} m n}(h) \overline{\boldsymbol{N}}_{\mathrm{o} m n}^{\prime}(-h)\right\}
$$

where the coefficients $\alpha$ and $\beta$ are to be determined. Substitution of (24) into (10) shows that

$$
\alpha=\beta=\frac{1}{\kappa^{2}-k^{2}}
$$

which determines completely the integral representation of the Green's function. The integration with respect to $h$ can be performed through the Cauchy integral theorem which gives

$$
\begin{gathered}
\overline{\overline{\boldsymbol{G}}}(b)\left(\overline{\boldsymbol{R}} \mid \overline{\boldsymbol{R}}^{\prime}\right)=\frac{\mathrm{i}}{x_{0} y_{0}} \sum_{m} \sum_{n} \frac{\left(2-\delta_{0}\right) a^{2}}{k_{c}^{2} k_{g}}\left\{\overline{\boldsymbol{M}}_{\mathrm{e} m n}\left( \pm k_{g}\right) \overline{\boldsymbol{M}}_{\mathrm{e} m n}^{\prime}\left(\mp k_{g}\right)+\right. \\
\left.\quad+\frac{a^{2} k^{2}}{a^{2} k^{2}+(1-a) k_{c}^{2}} \overline{\boldsymbol{N}}_{\mathrm{o} m n}\left( \pm k_{g}\right) \overline{\boldsymbol{N}}_{\mathrm{o} m n}^{\prime}\left(\mp k_{g}\right)\right\} \text { for } z \gtrless z^{\prime}
\end{gathered}
$$

where

$$
k_{g}^{2}=a^{2} k^{2}-a k_{c}^{2}
$$

\section{§ 4. Waveguide of circular cross-section}

In cylindrical coordinates the function $\psi(h)$ takes the form

$$
\psi_{\mathrm{e}_{n} \eta}(h)=J_{n}(\eta r) \underset{\sin }{\cos } n \phi \mathrm{e}^{\mathrm{i} h z}
$$

or

$$
\psi_{\mathrm{e}_{n} \xi}(h)=J_{n}(\xi r){ }_{\sin }^{\cos } n \phi \mathrm{e}^{\mathrm{i} h z} .
$$

The boundary condition in turn defines the value of $\eta$ and $\xi$ according to

$$
\frac{\partial J_{n}\left(\eta r_{0}\right)}{\partial r_{0}}=0
$$

or

$$
J_{n}\left(\xi r_{0}\right)=0
$$

where $r_{0}$ denotes the radius of the guide. Thus the vector wave functions for this configuration are $\overline{\boldsymbol{M}}_{\mathrm{e}_{\boldsymbol{0}} \eta \eta}$ and $\overline{\boldsymbol{N}}_{\mathrm{o}_{n \xi}}$ defined in (13) and (14). The orthogonality relationships of interest are

$$
\iiint \overline{\boldsymbol{M}}_{\mathrm{o}_{\tilde{o}^{n \eta}}}(h) \cdot \overline{\boldsymbol{N}}_{\mathrm{o}_{n^{\prime \prime} \xi^{\prime}}}\left(-h^{\prime}\right) \mathrm{d} v=0
$$


$\iiint \overline{\boldsymbol{M}}_{\mathbf{e}_{\mathbf{0}} \eta}(h) \cdot \overline{\boldsymbol{M}}_{\mathbf{e}_{\mathbf{o}^{\prime} \eta^{\prime}}}\left(-h^{\prime}\right) \mathrm{d} v=$
$=\left\{\begin{array}{l}0 ; \quad n \neq n^{\prime} \text { or } \eta \neq \eta^{\prime} \\ \pi^{2}\left(1+\delta_{0}\right)\left(r_{0}^{2} \eta^{2}-n^{2}\right) J_{n}^{2}\left(\eta r_{0}\right) \delta\left(h-h^{\prime}\right) ; \quad n=n^{\prime}, \eta=\eta^{\prime}\end{array}\right.$

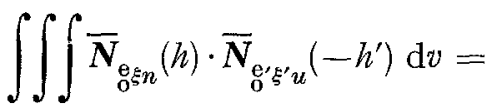

$=\left\{\begin{array}{l}\pi^{2} r_{0}^{2}\left(1+\delta_{0}\right) \frac{h^{2}+\xi^{2}}{h^{2}+a \xi^{2}}\left(\frac{\partial J n\left(\xi r_{0}\right)}{\partial r_{0}}\right)^{2} \delta\left(h-h^{\prime}\right) ; \quad n=n^{\prime}, \quad \xi=\xi^{\prime} \\ 0 ; \quad n \neq n^{\prime} \text { or } \xi \neq \xi^{\prime}\end{array}\right.$

The expansion of the Green's function is carried out in exactly the same fashion as for the rectangular case. Again a pole appears in the case of the $E$-mode wave and the integration path must be deformed in such a fashion as to eliminate the contribution. The final result in this case is

$$
\begin{aligned}
& \overline{\overline{\boldsymbol{G}}}(b)\left(\overline{\boldsymbol{R}} \mid \overline{\boldsymbol{R}}^{\prime}\right)=\frac{\mathrm{i}}{2 \pi} \sum_{n} a^{2}\left(2-\delta_{0}\right) \times \\
& \times\left\{\sum_{\eta=\eta_{m}} \frac{1}{k_{\eta}\left(r_{0}^{2} \eta^{2}-n^{2}\right) J_{n}^{2}\left(\eta r_{0}\right)} \overline{\boldsymbol{M}}_{\mathrm{e}_{\mathrm{o}} \eta \eta}\left( \pm k_{\eta}\right) \overline{\boldsymbol{M}}_{\mathrm{e}_{\mathrm{o}} \eta \eta}^{\prime}\left(\mp k_{\eta}\right)+\right. \\
& +\sum_{\xi=\xi_{m}} \frac{a^{2} k^{2}}{k_{\xi}} \frac{1}{\left[a^{\varepsilon} k^{2}+(1-a) \xi^{2}\right]} \cdot \frac{1}{r_{0}^{2}\left[\frac{\delta J n\left(\xi r_{0}\right)}{\partial r_{0}}\right]^{2}} \times \\
& \quad \times \overline{\boldsymbol{N}}_{\mathrm{e}_{\mathrm{o}} n \xi}\left( \pm k_{\xi}\right) \overline{\boldsymbol{N}}_{\mathrm{e}_{\mathrm{o}} n \xi}^{\prime}\left(\mp k_{\xi}\right) \text { for } z \gtrless z^{\prime}
\end{aligned}
$$

where

$$
k_{\eta}^{2}=\left(a^{2} k^{2}-a \eta^{2}\right), \quad k_{\xi}^{2}=\left(a^{2} k^{2}-a \xi^{2}\right) .
$$

\section{§ 5. Conclusion}

The method used in the derivation of the dyadic Green's function is useful in a wide variety of situations involving both moving and stationary media. Explicit results for the circular and rectangular cross section waveguides with a moving isotropic medium are derived in this paper. These functions are useful in the investigation of the fields arising from current and aperture sources in these waveguides. 


\section{Acknowledgement}

The research reported in this paper was sponsored by NSF Grants 10213 and 24102 and by NSF Science Faculty Fellowship 68196.

Received 23 December 1970

In final form 18 June 1971

\section{REFERENCES}

[1] Collier, J. R. and C-T TAI, IEEE Trans. MTT-13 (1965) 441.

[2] Du, L. J. and R. T. Compton, Jr., IEEE Trans. MTT-14 (1966) 358.

[3] Berger, H. and J. W. E. Griemsmann, IEEE Trans. MTT-16 (1968) 11.

[4] DALY, P., IEEE Trans. MTT-15 (1967) 274.

[5] Shiozawa, I., Proc. IEEE 54 (1966) 1984.

[6] Berger, H. and J. W. E. Griemsmann, Proc. IEEe 55 (1967) 1214.

[7]. Seto, Y. J., IEEE Trans. MTT-15 (1967) 455.

[8] TAI, C-T., Appl. Optics 4 (1965) 1347.

[9] Sommerfield, A., Partial Differential Equations, Academic Press, New York 1949.

Note added in proof: For a general discussion of the dyadic Green's function technique, the reader is referred to a recent book written by one of the authors (C-T. T.) entitled "Dyadic Green's Functions in Electromagnetic Theory", published by International Textbook Company Scranton, Pa., U.S.A. (October 1971). 